\title{
O MÉTODO DE PONTOS
}

\author{
Waldir Mantovani' \\ Fernando Roberto Martins ${ }^{2}$
}

\begin{abstract}
RESUMO - O método de pontos foi usado em diversas situações, comparado com outros métodos, modificado e amplamente discutido e criticado, podendo-se ter através da revisão bibliográfica uma visão de suas vantagens e limitaçōes, salientando-se naquelas a sua rapidez, precisão e pouca perturbação causada na vegetação e nestas, o diâmetro da agulha e a ação do vento.

Neste trabalho está sendo introduzido e discutido um novo índice, denominado índice de cobertura (IC), obtido da soma dos valores absolutos de freqüência, que representa a cobertura e de vigor, associado à biomassa.
\end{abstract}

Palavras-chave: fitossociologia, método de pontos.

ABSTRACT - The point method has been utilized in many situations, with or without modifications; it has also been compared to other methods and critically discussed. A review of the literature reveals the advantages (speed precision, minimum disturbance of the stand) and disadvantages (dependence on diameter of the needle, wind action).

A new index is introduced and discussed. It is referred to as the cover index (CI) and is obtained by summing absolute frequency, which represents cover, and absolute vigor, which is associated with biomass.

Key words: phytosociology, point method.

\section{Introdução}

A fitossociologia teve um excepcional desenvolvimento no Brasil na última década, principalmente pela escola iniciada na Universidade de Campinas, estado de São Paulo, tendo-se enfatizado os estudos do componente arbóreo de florestas e do cerrado.

O componente herbáceo, em geral, não recebeu o mesmo tratamento, em parte porque os estudos dos métodos fitossociológicos para sua amostragem não receberam a mesma atenção dada àqueles usados para a análise do com-

1 - Depto Ecologia Geral, IB/USP. Cx. P. 11461. CEP 05499, SP - SP. Bolsista do CNPq, processo n- 300304/81 - ZO07.

2 - Depto Morfologia e Sistemática Vegetais, IB/UNICAMP. 
ponente florestal de diversas formações vegetais (Mantovani, 1987).

Neste trabalho pretende-se divulgar um método fitossociológico desenvolvido para o estudo de espécies herbáceas, o método de pontos, através de revisão bibliográfica. Nesta revisão, que propõe apresentar os trabalhos mais relevantes, buscar-se-á indicar o seu desenvolvimento e as suas variadas aplicações. Revisões parciais anteriores foram efetuadas por Cain \& Castro (1959), Goodall (1952), Greig-Smith (1964), Hanson (1950a) e por West (1937).

\section{Resultados e discussão}

\section{Denominação do método}

Desde a sua primeira referência, o método vem recebendo diferentes denominações: "point quadrat" (Levy \& Davies, 1929), "point method" Lindquist, 1931), "point sampling" (Osborn et al., 1935), "point intercept" (Whitman \& Siggeirson, 1954), "point plot" (Heady, 1957), "stave point" (Müller, 1963), "point frame method" (Nermey, 1960) e "point sample" (Yarranton, 1966). No Brasil, Buselato \& Bueno (1981) o denominaram de "método de agulhas". Pela independência do mecanismo de amostragem, sugere-se o uso do termo "método de pontos" como o mais amplo para referí-lo, embora empreguem-se no texto, os termos: método de pontos, método de varetas, método de pinos, método de agulhas e amostra de pontos.

\section{História do método}

A primeira referência ao método de pontos foi feita por Cockaine (1926), quando alertou para a importância do estudo da relação entre o espaço ocupado e o número de indivíduos de uma espécie vegetal, ao examinar uma formação campestre na Nova Zelândia: "To obtain data as to the space occupied e method was divised, considered by teacher D.M.Y. Sommerville, of Victoria University College (Wellington) to be mathematically sound but, as it has not been sufficiently tested, no more need be said"'.

O uso do método foi indicado pela primeira vez por Levy (1927a) ao buscar informações sobre as espécies ocorrentes numa pastagem, visando um manejo econômico: "Cada ponto é analisado estrato por estrato, e cada espécie cobrindo o ponto da superfície do solo examinado, é anotada". Em sua análise apresentou o número médio de toques por cem pontos examinados. Em trabalhos posteriores, foram consideradas também, a porcentagem do solo descoberta (Levy, 1927b; Levy \& Smith, 1929), a porcentagem do solo coberta pela espécie em estudo (Levy \& Davies, 1929) e o número médio de toques 
obtido por cem pontos (Levy \& Davies, 1930).

Du Rietz (1930 apud Levy \& Madden, 1933) descreveu o método, salientando sua elevada exatidão, a pequena perturbação causada na vegetação e o menor tempo gasto na sua aplicação, quando comparado com outros métodos. Concluiu com uma expectativa favorável ao método de pontos.

Uma proposta de mudança na aplicação do método foi feita por Lindquist (1931), que escreveu: "No lugar da estrutura linear com 10 varetas usadas por Cockaine e Levy, foi empregada uma estrutura quadrada de $1 \mathrm{~m}$ por $1 \mathrm{~m}$ com 100 varetas igualmente espaçadas entre si". Esta foi a primeira referência, encontrada na literatura, ao aparelho usado para a aplicação do método. Além disso, esse autor teceu considerações sobre a influência do diâmetro da agulha usada nos resultados obtidos.

O método de agulhas foi descrito em detalhes por Levy \& Madden (1933): "Este método é baseado no conceito matemático de homogeneidade de uma área, igualmente representada por um ponto. Tendo-se um número suficiente de pontos amostrados, um quadro elaborado pode ser reproduzido. (...) $\mathrm{O}$ método consiste em amostrar um número de pontos ao acaso e anotar todas as espécies que são tocadas quando o ponto, simulado com o uso de uma vareta, é projetado através da relva. O instrumento usado é composto por uma estrutura conduzindo uma fileira de varetas de aço separadas duas polegadas entre si, sustentadas de tal forma que podem deslizar verticalmente num determinado trajeto. Essa estrutura é apoiada em suportes finos para facilitar a descida das agulhas até o solo. (...) Para amostrar somente as espécies dominantes da vegetação de um pasto, 100 pontos são suficientes, mas onde também for necessário amostrar os componentes mais raros, são precisos 400 a 500 pontos. (...) $\mathrm{Na}$ exposição dos resultados das análises quatro parâmetros estão envolvidos: (1) a porcentagem do solo coberto por uma espécie; (2) a porcentagem de cobertura de cada espécie; (3) a freqüência relativa de cada espécie e (4) a porcentagem de contribuição de cada espécie na vegetação.

Osborn et al. (1935) aplicaram o método na vegetação da Reserva Koonamore, Austrália, tendo obtido resultados insatisfatórios para áreas áridas, com vegetação aberta.

Num estudo efetuado em formações campestres no sul da Austrália, Crocker \& Tiver (1948) amostraram 300 a 500 pontos, tendo determinado que a uniformidade de tratamento foi o fator mais importante para a análise.

Arnborg (1949) usou a amostra de pontos na Suécia, considerando-a adequada para levantamentos rápidos em vegetação campestre.

Para Goodall (1952), apenas a porcentagem de solo coberto por uma espécie e a porcentagem de contribuição de cada espécie na vegetação possuem valor para análise da vegetação e propôs um novo parâmetro, que chamou de "cover repetition".

Para o cálculo da densidade das espécies amostradas pelo método, Whitman \& Siggeirson (1954) propuseram um parâmetro considerando o total de 
toques na espécie e a porcentagem de solo descoberto. As áreas basais de diversos espécimes de Gramineae foram obtidos através do método de pontos por Robinson (1954). Para a medição da altura das plantas, Heady (1957) propôs a aplicação da amostra de pontos, enaltecendo sua objetividade e facilidade de operação.

No seu estudo, Hurd (1961) usou o método, anotando o primeiro toque e o toque dado na base da planta, tendo calculado a porcentagem de cobertura, a constância, a freqüência relativa e a percentagem de contribuição de cada espécie na vegetação.

Num estudo comparativo entre uma área sujeita a pastoreio e outra, sem perturbações, Kleiner \& Harper (1972) utilizaram-se do método de varetas para a obtenção da cobertura. Também para o exame do efeito da pastagem de ovelhas sobre a relva, Cadwalladr \& Morley (1973) utilizaram o método de agulhas.

No Brasil, Bueno et al. (1979) e Buselato \& Bueno (1981) apresentaram trabalhos utilizando o método de pontos, associando-o ao de Braun-Blanquet, para estudos de áreas de campos, no Rio Grande do Sul.

Castellani (1986) estudou uma floresta secundária em Campinas, SP, através da aplicação de 420 pontos de amostragem fixos.

Mantovani (1987) empregou 300 pontos isolados em cada uma das cinco fisionomias de cerrado na Reserva Biológica de Moji-Guaçu e em três diferentes em Itirapina, estado de São Paulo.

Fabris et a l (1990) também amostraram 300 pontos em área de restinga, em Guarapari, ES.

\section{Variaçōes no aparelho para amostragem em seqüência}

A primeira modificação no aparelho usado para a aplicação do método de pontos foi sugerida por Lindquist (1931), que usou a mesma barra indicada por Levy \& Madden (1933), sobre um quadrado de $1 \mathrm{mx} 1 \mathrm{~m}$, em cima do qual podia ser deslocada lateralmente, sendo amostrados 100 pontos em cada local de amostragem.

Outro aparato que permitia movimentos na barra com 10 pinos foi proposto por Fenton (1933). A barra era fixa num eixo suporte e podia movimentar-se vertical e horizontalmente.

West (1937) simplificou o aparato e usou uma barra com 10 pregos fixos. Julin (1948) utilizou o aparelho modificado por Lindquist, na Suécia.

No estudo de uma área de "prairie" canadense, Coupland (1950) usou uma barra de $10 \mathrm{~m}$, com 10 agulhas, que denominou de "point transect", e obteve, através da amostragem, a área basal e a porcentagem de contribuição de cada espécie na vegetação.

Preocupado com a heterogeneidade das pastagens do Quênia e com a ne- 
cessidade de efetuar-se um grande número de amostras para analisá-las, Robinson (1955) sugeriu um aparato que permitia a variação entre os pinos e comparou o uso de agulhas a 6 e a 2 polegadas entre si, não obtendo diferença entre os resultados. Um aparelho que permitia a aplicação das agulhas sem que ocorressem movimentos laterais, que controlava o movimento vertical e que permitia a amostragem inclinada foi desenvolvido por Heady \& Rader (1958).

Ao invés do uso de barras horizontais, Nermey (1960) desenvolveu um aparato adaptado a uma roda de bicicleta, com 10 varetas perpendiculares ao eixo central, que o autor indicou para o estudo de campos com gramíneas baixas.

Müller (1963) usou um tubo em cujo interior passava uma vareta que podia, através de um eixo, ter a sua altura regulada e que permitia a amostragem a distâncias variáveis entre si. Foi denominado "stave point apparatus" e, de acordo com o autor, era uma adaptação do aparato apresentado por Tidmarsh \& Havenga (1955 apud Müller, 1963), que não permitia a regulação da altura.

Um aparato com 5 agulhas foi proposto por Radcliffe \& Mountier (1964a). As agulhas eram presas por molas que permitiam a retração. Broembsen (1965) descreveu um aparelho semelhante ao de Nermey (1960), que achou adequado para o estudo de áreas de savanas aberta e semi-aberta na África.

Uma adaptação do método de agulhas que permitisse a análise de vegetação herbácea densa, através de transeções lineares, foi desenvolvida por Long et al. (1972). Constituia-se de uma barra de duralumínio de $2,4 \mathrm{~m}$ de comprimento e seção retangular de $10 \mathrm{~cm} \times 1,8 \mathrm{~cm}$, presa nas extremidades por suportes que permitiam a regulagem da altura. A barra apresentava 80 furos pequenos, espaçados $2,5 \mathrm{~cm}$ entre si, através dos quais eram passadas agulhas de $0,5 \mathrm{~mm}$ de diâmetro e $16 \mathrm{~cm}$ de comprimento, conduzidas por uma bainha e capazes de retração regulada por um sistema de molas.

A distribuição de líquens no córtex de árvores foi estudada na Coréia, por Kim \& Lee (1975), que reduziram o tamanho do aparato para $10 \mathrm{~cm}$ de comprimento e o aplicaram, aleatoriamente, sobre os troncos de diversas espécies, tendo obtido a freqüência e a densidade das espécies.

Castellani (1986), utilizando um pino de $2 \mathrm{~m}$ de altura amostrou uma área de floresta em sucessão secundária em Campinas, SP, com o auxílio de um suporte de madeira adaptado à altura do trecho de vegetação amostrado.

\section{Mudanças no método:}

\section{Aplicação de um único ponto}

Blackmann (1935) sugeriu que o uso da amostragem por varetas agrupadas poderia ser desvantajoso no caso de uma espécie com distribuição conta- 
giosa, o que levaria a resultados tendenciosos. Recomendou o uso de pontos isolados e afirmou que o erro no uso de pontos juntos aumenta quando a vegetação não é homogênea e é menor quando todas as espécies distribuem-se ao acaso.

Através de uma correlação teórica simples, Rothery (1974) demonstrou ser possível aumentar a precisão na estimativa da cobertura vegetal, reduzindo o númiero de pinos no aparelho e mantendo o número de amostras. Concluiu pela ineficiência do uso de 10 agulhas no equipamento de amostras.

Para a análise das plantas invasoras da cultura do chá, no Ceilão, Eden \& Bond (1945) utilizaram-se da amostra de 100 pontos isolados. Estimaram as distâncias visualmente e efetuaram duas amostragens, uma em julho e outra em agosto. Introduziram uma modificação na amostra de pontos denominada de espeto ("skewer"), uma vareta de metal que era baixada na vegetação pela mão do observador.

Utilizando-se do mesmo método anterior, Bond (1947) concluiu que a porcentagem total de cobertura obtida tinha uma alta correlação com o peso seco das invasoras herbáceas.

$\mathrm{O}$ uso de agulhas isolada sé melhor que aquelas dispostas em estruturas de 10, para evitar-se a amostra de plantas com distribuição contagiosa, sendo necessário 1/3 do total dos pontos usados em seqüência, para obter-se igual resultado (Goodall, 1952). Para um inventário da cobertura herbácea e discussão da correlação com o tipo de solo, cobertura vegetal arbórea, declividade e outros fatores do meio, Evans \& Merton-Love (1957), na Califórnia (EUA), criaram o método de ponto do passo ("step point method"). Consistia na aplicação de uma única agulha guiada por um chanfro definido, na ponta da bota do pesqisador. A vareta ficava inclinada, aproximadamente a $30^{\circ}$ do solo, para que não houvesse perturbação na vegetação ao redor da amostra. Anotavam a primeira espécie tocada. Caso nenhum espécime fosse tocado, o pino era girado na direção de $180^{\circ}$ (qualquer sentido) e a primeira planta tocada era, então, anotada. Não amostravam o solo descoberto e, para a estimativa do total da cobertura, usaram um método visual.

Ripley et al. (1960) sugeriram o uso de amostras permanentes para o estudo de mudanças na vegetação e apresentaram um equipamento capaz de, rápida e acuradamente, recolocar amostras de pontos unitários.

Estudando a diversidade de pássaros, MacArthur \& MacArthur (1961) avaliaram as correlações existentes com a composição das plantas e com o dossel. Para a estimativa da área foliar das árvores usaram um arame rígido, anotando as folhas tocadas e, para o dossel, utilizaram um tubo de 10 pés de comprimento e 1,25 polegadas de diâmetro, como mira.

No uso do método de pontos para a estimativa da porcentagem de cobertura, apenas a presença ou a ausência da espécie é obtida em cada ponto. Para isto, Winkworth \& Goodall (1962) desenvolveram o tubo de mira de arames cruzados ("crosswire sighting tube"), composto por um tubo em cujo interior 
encontravam-se dois fios de arame cruzados perpendicularmente, formando um ponto de diâmetro pequeno.

Tentando evitar os erros decorrentes do uso de varetas com diâmetros que superestimam a amostra de pontos, Wilson (1963b) construiu um aparelho com uma agulha de diâmetro reduzido. Morrisson \& Yarranton (1970) apresentaram um aparato semelhante ao de Winkworth \& Goodall (1962), indicando-o para amostras verticais ou inclinadas. As maiores limitaçōes para o uso do aparelho foram o movimento na vegetação para a observação dos estratos inferiores e a colocação do aparato sobre arbustos densos.

Para vegetações herbáceas, altas e densas, foi usada uma lâmina de baioneta de $65 \mathrm{~cm}$ de comprimento, $4,5 \mathrm{~cm}$ de largura e $2 \mathrm{~mm}$ de espessura, anotando-se todos os toques das plantas na face cortante da lâmina. De acordo com Poissonet et al. (1972), um observador treinado poderia efetuar cerca de 350 observações num dia.

Amostras do conteúdo estomacal e de fezes de zebra foram analisadas através do método de pontos aplicado com o auxílio de um microscópio (Owaga, 1977).

Reynolds \& Edwards (1977) desenvolveram um telescópio de foco curto ("short focus telescope") para a análise da cobertura do solo. De acordo com os autores, ofereceu uma estimativa de cobertura acurada e relativamente rápida, não apresentou dificuldades de uso e causou um pequeno erro de paralaxe, tendo as vantagens do tubo de mira de arames cruzados de Winkworth \& Goodall (1962) e cobrindo as suas deficiências. Consideraram-no, ainda, adequado para a análise de diferentes níveis de vegetação.

Os organismos existentes na zona de entre marés em nove estações do porto de Long Beach, Califórnia, foram amostrados por pontos aleatórios (Loi, 1981).

Castellani (1986) em seu estudo da sucessão secundária inicial em uma área de mata tropical semi-decídua, efetuada em Campinas, SP, aplicou um total de 420 pontos aleatórios ao longo de transecções previamente demarcadas, em cada uma das dezesseis etapas amostrais realizadas. Por objetivar o estudo de variações temporais na estrutura florística, os pontos amostrais foram fixados com o uso de palitos de madeira coloridos.

Para o estudo das variações estruturais do componente herbáceo-subarbustivo do cerrado, Mantovani (1987) utilizou uma agulha de metal de $3 \mathrm{~mm}$ de diâmetro e $1,1 \mathrm{~m}$ de comprimento, solta a cada três passos, tendo anotado a espécie, o número e a altura dos toques.

Fabris et al. (1990) amostraram 300 pontos na restinga de Setiba, litoral sul do estado do Espírito Santo, utilizando uma agulha de $1,8 \mathrm{~m}$ de comprimento e $3 \mathrm{~mm}$ de diâmetro, disposta ao longo de linhas que cortavam a vegetação. 


\section{Inclinação na amostra}

$\mathrm{Na}$ análise que fizeram sobre diverśos métodos usados para o estudo de pastagens, Tinney et al. (1937) propuseram a inclinação da vareta representativa do ponto em $45^{\circ}$. Para esses autores, o método de pontos inclinados apresentava vantagens em relação ao uso de agulhas verticais, pela maior constância com que eram amostradas, neste, as plantas de folhas largas. Além disso, o pino inclinado podia ser mais facilmente visto pelo observador, cobria uma área maior por leitura, anotando um número maior de plantas e, por isto, incrementando a precisão.

Utilizando agulhas inclinadas Arny \& Schmid (1942) concluíram serem necessários fatores de correção para correlacioná-lo com o peso e alertaram para a necessidade de considerarem-se fatores, como mudanças sazonais e na composição da vegetação estudada. Para Goodall (1952) a inclinação da agulha em $45^{\circ}$, para o etudo de uma vegetação com um determinado número de pontos, aumenta a precisão mas superestima os dados.

Wilson (1959) propôs o uso conjunto de amostras de pontos verticais e horizontais para a estimativa do ângulo e da densidade da folhagem. Apresentou um aparato capaz de efetuar a amostragem com um único ponto por amostra.

Para aquele autor (Wilson, 1960), de acordo com estudos teóricos por ele efetuados, a freqüência relativa resultante do ângulo de inclinação das folhas é maior na amostra vertical, reduz-se a $45^{\circ}$ e é menor em $32,5^{\circ}$. Correlacionou o ângulo da mostra com o ângulo das folhas.

A estimativa da produtividade de ervas foi feita por Hughes (1962) com 200 amostras de pontos inclinados. Ocorreu uma baixa correlação dos dados obtidos com o corte e a secagem de 20 amostras da vegetação. Discutiu diversos fatores que poderiam ter influído nos resultados.

Reppert et al. (1962) estudaram a produtividade de um pasto anual, na Califórnia, com um aparelho com 30 pinos inclinados em $45^{\circ}$, anotando o primeiro toque e a altura da planta tocada.

Estimativas do ângulo e da densidade da folhagem de uma espécie vegetal puderam ser feitas através do uso de varetas inclinadas (Wilson, 1963a).

Estudando a disposição espacial da folhagem, Philip (1965) aplicou o r. étodo de agulhas inclinadas, fez análises matemáticas e discussões críticas a respeito e concluiu que, apesar de exigir um grande número de amostras, o método é eficiente, relativamente simples e capaz de fornecer dados consistentes.

O refinamento das técnicas de amostras por pontos, ainda segundo Philip (1966), conduziu a três equações integrais. Neste seu trabalho, além de tê-las discutido, o autor apresentou asspectos estatísticos da amostragem pelo método e debateu a inclinação dos pinos em vários ângulos para estudos da distribuição espacial da folhagem. 
Miller (1969) estimou o ângulo de inclinação das folhas em diversas espécies de uma floresta, para testar modelos de passagem da radiação solar através do dossel, de acordo com o método de Wilson (1959).

Também para o estudo do dossel de uma floresta, Ford \& Newbold (1971) utilizaram o método de varetas inclinadas, tendo usado uma vara de metal de $0,5 \mathrm{~mm}$ de diâmetro e inclinações a $0^{\circ}, 32,5^{\circ}$ e $90^{\circ}$. Concluíram que a folhagem tende a se agregar em diferentes padrões que refletem o desenvolvimento da estrutura dos ramos. $O$ índice de área foliar, o ângulo e os padrões de agregação das folhas de sete clones de chá foram obtidos através do método de agulhas inclinadas, tendo sido usada uma agulha de metal de $3 \mathrm{~mm}$ de diâmetro, em ângulos de $0^{\circ}, 32,5^{\circ}$ e $90^{\circ}$. O índice de área foliar foi comparado com o obtido por métodos destrutivos e o ângulo foliar foi confrontado com medidas diretas. Houve correlação positiva entre os dois métodos usados para o índice de área foliar, não encontrada para os métodos de ângulo foliar. Os dados revelaram diferenças significativas nas características das folhas e do dossel dos clones estudados (Magambo, 1977).

Fowler \& Arvanitis (1981) indicaram um procedimento para eliminar os desvios estatísticos que surgem do método de varetas horizontais tiradas da margem de florestas.

\section{Uso de grades}

Stanton (1960) propôs um aparato para a amostragem visual do ponto, que consistia de um quadrado de madeira em cujo interior eram passados, paralelos e perpendicularmente, vários fios de arame que formavam os pontos, nos seus cruzamentos. Este conjunto era suportado por pernas de metal, reguláveis a alturas desejadas. Para o estudo autoecológico de Acacia karoo, efetuado na África do Sul, Du toit (1968) estimou a área foliar através de uma adaptação do método de pontos. Traçou uma malha de linhas finas, distantes $8 \mathrm{~mm}$ e formando ângulos retos entre si, numa folha de papel duro, branco, e obteve 1.521 pontos de interseção numa área de $924 \mathrm{~cm}^{2}$. Retirou os foliólulos de uma folha e os espalhou sobre a malha, evitando a sobreposição. Os pontos de interseção da malha serviram como pontos de amostras e o número deles, coberto pelos foliólulos, pode ser estabelecido. Calculou a área foliar através da relação: (№ de pontos coberto/NNo de pontos amostrados) x Área amostrada.

Um quadrado de $1 \mathrm{~m} \times 1 \mathrm{~m}$ contendo uma grade de 100 pontos foi utilizado, no Ceilão, para medir a recorbertura de campos baixos após o pastejo de elefantes (Mueller-Dombois \& Cooray, 1968 apud Mueller-Dombois \& Ellenberg, 1974).

Uma discussão sobre as várias técnicas empregadas para a amostragem, em estudos de plantas fósseis, foi feita por Scott (1977). Neste trabalho o autor empregou para a amostra de fósseis, em meio metro quadrado de rocha, 
uma malha de 100 pontos. O método forneceu o número de fragmentos e a área coberta por espécie.

Garrison (1949) propôs o uso de um aparelho cujo princípio de amostragem era semelhante ao das grades. Era usado para a análise da cobertura do dossel de florestas e assemelhava-se a um periscópio, permitindo a amostragem de 25 pontos de cada vez. Os pontos, eqüidistantes, eram marcados em folha de plástico.

\section{Comparação com outros métodos}

O método de pontos foi considerado mais exato, mais rápido e capaz de perturbar menos a vegetação que os métodos de linha, de parcelas e de faixas (Du Rietz, 1930 apud Levy \& Madden, 1933).

Davies (1933) apontou sete métodos para a análise quantitativa de pastagens usados até aquela data: (1) freqüência específica, (2) mapeamento, (3) porcentagem de área ou porcentagem de solo coberto, (4) método de pontos, (5) porcentagem de freqüência, (6) porcentagem de produtividade e (7) métodos de estimativa em escala de 0 a 10 (porcentagens de freqüência, de produtividade e de cobertura).

Salientou como vantagens de método de pontos a exatidão com que se definem o ponto e o toque nas espécies.

Hanson (1934) comparou os métodos de listagem por área, contagem e listagem, listagem e pesagem, pontos, freqüência-abundância e mapeamento. Concluiu que o mapeamento era demorado, que o método de listagem por área e de contagem e listagem eram indicados para amostras permanentes e forneciam dados para análise estatística, conduzindo à formulação de conclusões mais definitivas e confiáveis que os métodos de pontos e de freqüênciaabundância. Estes, entretanto, forneceram valiosos dados adicionais, principalmente pela rapidez do seu uso. Indicou para as pesquisas de reconhecimento e para análises da relação solo-planta, nos problemas de erosão, uma conjunção dos métodos de pontos e de mapeamento.

O método de agulhas usado por Lindquist (1931), foi comparado com o método de freqüência de Raunkiaer (1934), tendo Bocher (1935) deduzido que ambos se completavam.

Para a comparação de seis diferentes métodos de análise de pastagens, Tinney et al. (1937) montaram experimentos em dois campos. Foram testados os seguintes métodos: de linhas, de freqüência específica, de porcentagem de freqüência, de porcentagem por área, de pontos verticais e de pontos inclinados em $45^{\circ}$. O método de pontos verticais foi aplicado de duas maneiras distintas: a primeira considerando-se um único toque por agulha e a outra considerando todos os toques até o solo. A primeira forma foi desvantajosa por tocar mais vezes as plantas altas. Os métodos de pontos verticais, consideran- 
do-se todos os toques, e o de pontos inclinados foram considerados mais precisos e capazes de fornecer um número maior de dados que os demais. A amostra inclinada foi considerada melhor que a vertical, por ser mais fácil de ser obtida e por ser mais precisa.

Ao comparar os métodos de agulhas e de porcentagem por área, West (1937) concluiu que aquele superestimou a importância de algumas espécies.

Charpentier \& Saarela (1941) indicaram o método para investigações na vegetação em pastagens e o consideraram mais objetivo e mais preciso que outros métodos rápidos.

Para o cálculo da porcentagem de cobertura de cada espécie, usando o método de varetas inclinadas, Henson \& Hein (1941) incluíram, no divisor, os pontos em branco encontrados. O resultado obtido foi semelhante àquele conseguido pelo método de porcentagem por área.

Uma comparação entre três métodos para a análise de vegetação de gramíneas baixas foi feita por Ellison (1942). Cinco observadores treinados aplicaram os métodos de mapeamento com pantógrafo, de listagem e densidade e de pontos, quatro vezes em cada uma de três áreas escolhidas. Em todos os métodos a diferença entre observadores foi maior na área com maior densidade e maior número de espécies. $\mathrm{O}$ mapeamento com pantógrafo requereu uma área 50\% maior, foi menos consistente que o de listagem e densidade em todas as áreas, mais consistente que o de pontos nas áreas de baixa e média densidades e foi o mais demorado. O método de listagem e densidade necessitou da mesma área que o de pontos, apresen bu resultados mais consistentes que os demais, exceto o de pontos na área de alta densidade e requereu o mesmo tempo que este. O método de pontos poderia ter aumentada a sua eficiência com um número maior de projeções. Em vista dos resultados sugeriu o método de listagem e densidade para o estudo de vegetação de gramíneas baixas.

Os resultados obtidos por Drew (1944) em Missouri, EUA, ao efetuar comparações entre métodos de análise de pastagens em uma vegetação mista, tendo como parâmetro o método de listagem-pesagem, levaram-no a concluir que: o método de pontos inclinados forneceu resultados mais satisfatórios que o método de listagem e contagem; a consideração de todos os toques, até o solo, foi melhor que a análise do primeiro toque; não houve necessidade do uso de fatores de ajuste para correlacioná-lo com o peso seco.

Ao comparar o método de pontos inclinados com a separação manual, para o estudo da porcentagem da composição de uma associação de grama azul e de trevo branco, Sprague \& Myers (1945) concluíram que a amostra de pontos forneceu uma medida real para a porcentagem de trevo branco, mas que os dados para a grama azul eram superestimados.

Ahlgren (1947) apresentou uma relação de 32 métodos para estudos da composição florística de pastagens e afirmou serem os vários métodos similares, modificações ou combinações dos métodos de freqüência específica, de 
mapeamento, de porcentagem, de freqüência, de porcentagem da área ou do solo coberta, de pontos, de porcentagem de produtividade ou dos métodos de estimativa numa escala de 0 a 10 . Concluiu que nenhum método pode ser usado satisfatoriamente em todas as condições, nem possuem todas as qualidades desejadas.

Para Crocker \& Tiver (1948), o método de pontos é mais objetivo e rápido que outros métodos que apresentam igual segurança. Numa comparação entre o método de pontos inclinados, separação manual e estimativa visual para cálculo da porcentagem da composição, Leasure (1949) concluiu que os três dão resultados semelhantes.

Hanson (1950a) indicou o uso do método de pontos e o de listagem por área para estudos de cobertura, por serem mais objetivos que o método de listagem-densidade. Para esse autor foi possível, com o uso de fatores de correção no método de pontos, obterem-se dados próximos à porcentagem de peso, devendo-se evitar o uso de fatores constantes, dadas as variações no peso que ocorrem durante o ano (Hanson, 1950b).

Para Goodall (1952) os resultados obtidos com o emprego do método de agulhas inclinadas e verticais não foram idênticas, mas ambos válidos.

Comparando o método de intercepção linear com o de pontos, numa área de vegetação mista em Dakota do Norte, Whitman \& Siggeirson (1954) observaram que o método de pontos superestimou a densidade e subestimou a área basal.

Tendo apresentado um estudo da relação entre a orientação das folhas numa planta e a área da sua projeção, Winkworth (1955) demonstrou serem mais precisas as amostras obtidas com pontos isolados, efetuadas através de estimativa visual, que as obtidas com o uso de pinos em aparatos com 10 aguThas, e que as amostras inclinadas superestimam a cobertura I la vegetação em relação às amostras verticais.

Kemp \& Kemp (1956), na Inglaterra, compararam a aplicação do método com a estrutura de 10 pontos em 200 amostras, com aquele de 5, 2 e 1 ponto por amostra, para diversas espécies. Concluíram ser necessário aumentar o número de repetições quando diminui o número de pontos no aparelho.

O método do ponto-do-passo ("step point") de Evans \& Merton-Love (1957) gastou de 1/6 a 1/8 do tempo usado para a aplicação do método com um aparato de 10 agulhas. Este, entretanto, foi melhor para a amostra de espécies de ocorrência rara.

Johnston (1957) efetuou uma análise para indicar o tamanho, o número de amostras e o método mais eficiente entre a intercepção por linha, o método de pontos verticais e o método de linha de base, para estudos de campos em Alberta, Canadá. Obteve os seguintes resultados: o método de linha de base foi mais rápido, tendo detectado um número menor de espécies e fornecido dados mais variáveis; a intercepção linear tomou mais tempo, indicou o maior número de espécies e apresentou dados próximos aos do método de pontos 
que, por sua vez, apresentou a menor variação de dados. Considerou o método de pontos mais adequado para a vegetação estudada.

Ao efetuarem a comparação entre a separação manual com posterior obtenção do peso seco, usado como padrão, e os métodos de pontos inclinados, de pontos verticais e de estimativa visual da porcentagem da composição, Van Keuren \& Ahlgren (1957) concluiram que as correlaçōes eram maiores com os pontos inclinados; ocorreu uma grande variação entre a estimativa visual e os métodos de pontos; a análise de todos os toques forneceu uma variação menor que apenas o primeiro toque; os dois métodos de pontos apresentaram poucas diferenças entre si e em comparação com o método usado como padrão. No conjunto, o método de pontos inclinados foi melhor que os demais.

Numa comparação entre o método de linha de base ("loop method"), o método de pontos aplicados ao longo de uma linha e com um aparato de dez pontos, Cook \& Box (1961) concluíram que o último era mais preciso e mais rápido que os demais.

Reppert et al. (1962) utilizaram um aparelho com 30 pinos inclinados em $45^{\circ}$ para a estimativa da produtividade de campos na Califórnia. Compararam os dados obtidos com os resultados alcançados através de estimativa visual para altura e cobertura da vegetação.

Coeficientes de regressão simples foram estatisticamente significativos para altura, cobertura por estimativa visual, altura $\mathrm{x}$ cobertura por estimativa visual, altura $\mathrm{x}$ cobertura pelo método de pontos inclinados.

Utilizando-se 4 métodos para a estimativa da porcentagem de cobertura (mapeamento, estimativa visual, intercepção linear e pontos), Winkworth et al. (1962) deduziram que, exceto a intercepção linear, todos os métodos apresentaram resultados semelhantes quando aplicados em amostras circulares de $1,9 \mathrm{~m}$ de diâmetro, retangulares de $5 \mathrm{~cm} \times 2 \mathrm{~cm}$ ou de $10 \mathrm{~cm} \times 4 \mathrm{~cm}$, sendo o método de mapeamento o mais demorado e os de ponto e de estimativa visual em pequenos círculos os mais rápidos.

No Texas (EUA), Brun \& Box (1963) compararam a intercepção linear com a aplicação do método de pontos, utilizando um aparato com 10 varetas, para estudos em vegetação arbustiva e deduziram que o último foi mais rápido e tão ou mais eficiente, em alguns casos, que aquele.

Poissonet et al. (1972), ao desenvolverem o método de baioneta, compararam-no com o de pontos num aparato de 10 agulhas e obtiveram menor desvio para aquele.

As estruturas das populações de duas comunidades vegetais do sudoeste da Boêmia (Tchecoslováquia) foram estudadas através dos métodos de pontos com agulhas, de amostra visual, de peso seco, de área foliar, de número de i ndivíduos e de volume. As variações encontradas foram devidas ao diâmetro da agulha, à morfologia das folhas e à forma de crescimento das várias espécies. Kubíkova \& Rejmánek (1973) concluíram que as determinaçōes feitas com agulhas de pequeno diâmetro eram diretamente proporcionais ao peso seco e à 
área foliar com regressão significante a 0,01 e 0,05 , não sendo obtida nenhuma regressão significativa para volume e para o número de indivíduos. Apresentaram gráficos comparando os diferentes métodos usados.

Para Mueller-Dombois \& Ellenberg (1974) as análises de cobertura com a inclinação das agulhas levariam a um incremento no número de troques e superestimariam os resultados em relação ao uso de agulhas verticais, por haver um incremento no número de toques.

No estudo que fizeram do hábito alimentar de coiotes, Johnson \& Hansen (1977) compararam a amostragem por pontos e a separação manual de fragmentos de fezes. Ambos apresentaram resultados satisfatórios, sendo a amostra por pontos oito vezes mais rápida que a separação manual.

Na Polônia, Tomialoja (1977) comparou o método de mapeamento com os de transeção e de pontos para promover o censo de pássaros. Também para o censo de pássaros, na Colômbia, Green (1978) comparou os métodos de pontos e de transeção, tendo gasto duas a três vezes mais tempo para o primeiro método, exceto para Cebus e para Saguinus. Os dados de densidade foram mais consistentes para o método de transeção. Apareceram poucas discrepâncias entre os dois métodos para cebídeos, que regularmente utilizam o dossel superior.

Um método seqüencial de amostra de artrópodos que ocorrem no algodeiro foi comparado ao método de varetas, tendo sido gasto $76 \%$ do tempo usado por este e reduzida a área de amostragem em $86 \%$, tendo sido recomendado por Rothrock \& Sterling (1982) para esta amostragem.

Floyd \& Anderson (1987) compararam a intercepção linear, o método de pontos e a estimativa da cobertura, na estepe com predomínio de Artemisia tridentata (Compositae) em Idaho, sudeste dos Estados Unidos. O método de pontos apresentou menor grau de precisão que a linha, tendo gasto um terço do tempo de amostragem e foi o mais eficiente dos três métodos.

O método de pontos foi desenvolvido originalmente para estudos de cobertura, composição e de freqüência das espécies em pastagens naturais ou artificiais (Davies, 1933; Fenton, 1933; Goodall, 1952; Levy \& Madden, 1933).

Quando comparado com diversos outros métodos, que forneciam os mesmos parâmetros, mostrou-se, geralmente, mais rápido, capaz de causar menores perturbações e com, pelo menos, o mesmo nível de exatidão (Brum \& Box, 1963; Charpentier \& Saarela, 1941; Cook \& Box, 1961; Crocker \& Tiver, 1948; Du Rietz, 1930 apud Levy \& Madden, 1933; Goodall, 1952; Heady, 1957).

Devido a essas características, foi amplamente estudado e teve ampliadas as suas aplicações.

Assim, Yarranton (1966) propôs que, além de anotar-se a espécie tocada no ponto, também fosse anotada a espécie em contato com aquela, o mais próximo do ponto. Com esse dado o autor calculou os valores de $\mathrm{X}^{2}$ da associação interespecífica, que serviram para análise de associações. Essa análise 
foi discutida por Radclife \& Mountier (1964a) e por Morrison \& Yarranton (1970).

Para Kershaw (1973) o método permitiu a obtenção de padrões de distribuição das espécies.

Coupland (1950) e Robinson (1954) indicaram-no para fornecer a área basal das espécies.

Enquanto a agulha representativa do ponto desce verticalmente, pode tocar as espécies de uma vegetação em diversas alturas, fornecendo dados sobre a estratificação (Du Rietz, 1933 apud Levy \& Madden, 1933; Goodall, 1952). A partir desses dados, Poissonet (1971 apud Mueller-Dombois \& Ellenberg, 1974) e Daget \& Poissonet (1971 apud Mueller-Dombois \& Ellenberg, 1974) desenvolveram um método para o cálculo da biomassa. Para Mueller-Dombois \& Ellenberg (1974) a correlação entre a estratificação e a biomassa pode ser feita, empiricamente, para cada tipo de vegetação e, tal como a relação entre o volume da vegetação e o peso, varia de espécie para espécie, de lugar para lugar e no tempo.

Diversos autores obtiveram correlaçōes diretas entre o peso seco da vegetação e os dados obtidos através do método. Aplicando agulhas verticais, Levy \& Madden (1933) correlacionaram o peso seco com a porcentagem de contribuição de cada espécie para a composição da pastagem e Bond (1947) correlacionou-o com a porcentagem de cobertura.

Drew (1944) e Kubíkova \& Rejmánek (1973) aplicaram varetas inclinadas e correlacioanram o peso seco com a porcentagem de cobertura e com a porcentagem de contribuição, respectivamente. Mueller-Dombois \& Ellenberg (1974) recomendaram o método para obter-se a biomassa de plantas em comunidades herbáceas.

Charpentier \& Saarela (1941) e Crocker \& Tiver (1948) afirmaram não haver correlação entre os dados obtidos com o método de pontos e o peso seco. Outros autores também não obtiveram essa relação e propuseram o uso de fatores de correção, passíveis de serem usados com a porcentagem de contribuição na composição ou com a porcentagem de cobertura (Arny \& Schid, 1942; Arny, 1944; Hanson, 1950a; Hughes, 1962; Van Keuren \& Ahlgren, 1957). Esses fatores devem variar conforme as espécies, período do ano e o desenvolvimento da vegetação, como salientou Arny (1944), para quem o peso seco por toque, obtido na análise de uma pastagem mista, foi maior para a alfafa do que para as gramíneas e elevou-se com o aumento da altura da vegetação.

Para Radcliffe \& Mountier (1964a) o método de agulhas, verticais ou inclinadas, não é recomendado para a obtenção do peso nem do volume.

Estudos da inclinação, densidade, padrões de distribuição e área foliar foram realizados através do método, com o uso de agulhas inclinadas (Ford \& Newbold, 1971; Magambo, 1977; Miller, 1969; Philip, 1965; Wilson, 1959; 1960; 1963b; Winkworth, 1955). Mudanças florísticas e fitossociológicas que 
ocorrem numa vegetação ou variações sazonais de uma espécie podem ser detectadas pelo método. Todos os trabalhos que sugeriram o seu uso enfatizaram a necessidade de manterem-se as amostras fixas (Charpentier \& Saarela, 1941; Goodall, 1952; Mueller-Dombois \& Ellenberg, 1974). Ripley et al. (1960) chegaram a desenvolver um aparato capaz de permitir a re-amostragem de um determinado ponto.

O método de pontos, como os demais métodos, apresenta suas limitações e diversos fatores foram apontados como capazes de influir nos seus resultados:

- altura da vegetação. Entre diversos outros fatores capazes de influir nas análises realizadas numa pastagem, Crocker \& Tiver (1948) demonstraram preocupação com a altura da vegetação. O método de agulhas forneceu resultados mais satisfatórios em vegetação baixa.

Radcliffe \& Mountier (1964b), ao analisarem o efeito da altura da vegetação no uso do método, concluíram que o número de toques foi afetado sensivelmente por este fator; que pareceu haver uma relação linear entre o aumento na altura da vegetação para o primeiro toque, entre uma a quatro polegadas; que o efeito foi maior, considerando-se o total de toques ao invés de apenas o primeiro; que não houve coeficientes de regressão consistentes para qualquer espécie e que comparações entre diversas associações vegetais efetuadas pelo método de pontos só poderiam ser realizadas quando as vegetações tivessem a mesma altura.

- densidade da vegetação. Osborn et al. (1935) usaram o método de pontos em zonas áridas, com vegetação aberta, tendo-o abandonado por fornecer dados insatisfatórios. Isto vai de encontro aos trabalhos de Hanson (1938), para quem o método foi indicado para vegetação densa, e de Ellison (1942) que, ao compará-lo com outros métodos, concluiu ser menos eficiente que o "pantograph chart" e o "density list" em áreas de baixa e de média densidades, tendo apresentado resultados mais consistentes que os demais em áreas com alta densidade.

- morfologia e diferentes formas de vida. Para Drew (1944) a morfologia das espécies influiu nos resultados por ele obtidos, quando comparou o método de agulhas com diversos outros. Para analisar gramíneas que cresciam em tufos, Goodall (1953) sugeriu um tratamento matemático para a análise da cobertura média. Os erros obtidos na porcentagem de freqüência, quando da aplicação do método, foram dependentes da morfologia da folha e da forma de crescimento das várias espécies, variando de $70 \%$ em Polygonum hydropiper a $322 \%$ em Eleocharis ovata (Kubíkova \& Rejmánek, 1973).

- vento. O vento foi considerado por vários autores (Crocker \& Tiver, 1948; Morrison \& Yarranton, 1970; Mueller-Dombois \& Ellenberg, 1974) como um sério problema para o uso do método, seja com pinos ou com aparelhos que permitam a análise visual. Recomendaram a aplicação do método em períodos sem ventos, que permitam a observação clara dos toques. 
- diferentes observadores. Comr arando diferentes métodos de análise de vegetação e utilizando cinco observadores treinados, aplicando quatro vezes cada método, Ellison (1942) concluiu que a diferença entre observadores foi mais evidente em áreas com maiores densidades e emaranhado de indivíduos.

Da sua análise sobre a influência de diferentes observadores nos resultados obtidos com o método de pontos, Crocker \& Tiver (1948) afirmaram que a altura da vegetação entre 10 e $15 \mathrm{~cm}$ foi a que apresentou as menores variaçōes, as quais foram maiores em alturas superiores. Goodall (1952) reafirmou a diferença entre observadores e Mueller-Dombois \& Ellenberg (1974) atribuíram grande parte dos erros surgidos nos aparelhos óticos, usados por diferentes observadores, ao efeito de paralaxe.

- diâmetro da agulha. O método de pontos representa a máxima redução da área de amostragem (Goodall, 1952). O uso de agulhas ou pinos com diâmetros razoáveis superestimam os resultados obtidos, recomendando-se agulhas com o menor diâmetro possível (Goodall, 1952; Lindquist, 1933; Radcliffe \& Mountier, 1964a; West, 1937).

As plantas micrófilas são as que apresentam os maiores erros devidos ao diâmetro da agulha (Winkworth, 1955).

Alguns autores conseguiram quantificar a superestimativa causada. Para Wilson (1959), pinos de $2 \mathrm{~mm}$ de diâmetro superestimam em $5 \%$ a densidade de plantas com folhas de $4 \mathrm{~cm}$ de largura e em $200 \%$ a de plantas com folhas de $1 \mathrm{~mm}$ de largura. Para Ford \& Newbold (1971) o diâmetro de 0,5mm superestimou a cobertura em 1,25 a $0,56 \%$.

Quando a agulha usada para a análise com o método tem um diâmetro apreciável, causa superestimativa da área foliar. Um estudo teórico mostrou que dobrando-se o diâmetro da agulha ou reduzindo-se à metade a largura da folha, o erro na estimativa da freqüência relativa dobrou. Com as técnicas que vêm sendo usadas, os erros, freqüentemente, excedem $50 \%$ e a estimativa obtida usualmente é menos acurada que as estimativas visuais (Wilson, 1963b). De acordo com este autor, resultados suficientemente acurados podem ser obtidos com uma agulha estreita e aplicando-se fatores de correção. Pode-se evitar os erros considerando-se os toques efetuados pela ponta fina e não pelos lados da agulha, ao descer a vegetação. Pode-se calcular o erro por $\mathrm{E}=(100$ d) $/(\mathrm{c} . \mathrm{b}) \mathrm{x}(\mathrm{d}+\mathrm{c}+\mathrm{b})$, onde $\mathrm{E}=$ erro, $\mathrm{d}=$ diâmetro da agulha, $\mathrm{c}=$ comprimento da folha, $b=$ largura da folha. Para o cálculo da freqüência relativa (№ de contatos/100 amostras), para plantas de folhas longas e estreitas o erro se duplica a cada redução da largura foliar à metade ou duplicando-se o diamnetro da agulha.

Para plantas com folhas arredondadas, o erro é menos influenciado pela forma da folha do que pelo seu tamanho. A variação da relação c/b de 1 a 64 causa, aproximadamente, uma variação de três vezes para mais ou para menos no erro. Por outro lado, uma variação na largura de 1 a $30 \mathrm{~mm}$ causa, aproxi- 
madamente, uma variação de 50 vezes no erro, com a relação $\mathrm{c} / \mathrm{b}$ constante. Tem-se que dar maior atenção ao tamanho que à forma da folha.

A magnitude do erro esperado para agulhas de $2 \mathrm{~mm}$ é, freqüentemente, de $10 \%$, sendo comum obterem-se erros de $30 \%$ quando se analisam diversas espécies, e encontrarem-se erros que superam $100 \%$ para plantas micrófilas e gramíneas de folhas estreitas. Os erros para a estimativa da porcentagem de cobertura têm a mesma magnitude dos erros para freqüência relativa. A porcentagem de contribuição de cada espécie para a vegetação, ou seja (número de toques numa espécie/total de toques na vegetação)x100, será sub ou superestimada, conforme a espécie. Para a redução do erro na freqüência relativa pode-se multiplicá-la por um fator de correção $(c . b) /(c+d) \cdot(b+d)$, onde $c=$ comprimento da folha, $\mathrm{b}=$ largura da folha e $\mathrm{d}=$ diâmetro da agulha (Wilson, 1963b).

Outra análise detalhada acerca dos erros causados pelo diâmetro da agulha foi feita por Kubíkova \& Rejmánek (1973). Compararam agulhas de $0,1 \mathrm{~mm}, 4,5 \mathrm{~mm}$ de diâmetro e um método de estimativa visual. Para o cálculo da porcentagem de cobertura com o uso da agulha com $0,1 \mathrm{~mm}$ e através da estimativa visual obtiveram dados que mudaram substancialmente o grau de importância de uma espécie particular. Para os autores, apenas determinações efetuadas com agulhas de pequeno diâmetro são úteis para análise da estrutura de populações.

Para evitar que os dados fossem superestimados, foram desenvolvidos diversos aparatos para a amostragem ótica dos pontos (Goodall, 1952; Ibrahim, 1971; Kubíkova \& Rejmánek, 1973; Morrison \& Yarranton, 1970; Reynolds \& Edwards, 1977; Stanton, 1960; Winkworth, 1955; Winkworth \& Goodall, 1962).

- número de amostr as. O número de pontos requeridos para a análise de uma vegetação é variável com o tipo de vegetação e com a freqüência das suas espécies componentes (Goodall, 1952). As espécies raras necessitam de muitas amostras (Blackmann, 1935). Levy \& Madden (1933) indicaram a aplicação de 100 pontos para a análise das espécies dominantes de uma pastagem e de 400 a 500 pontos para amostras das espécies mais raras. Num estudo efetuado na Austrália, Crocker \& Tiver (1948) concluíram que 300 a 500 pontos amostrados foram insuficientes.

Para campos mistos, Whitmann \& Siggeirson (1954) propuseram a aplicação de 1.400 pontos, considerando-se todos os toques e 3.600 pontos, apenas para contatos basais.

Para Mueller-Dombois \& Ellenberg (1974) numa cobertura vegetal homogênea cerca de 200 pontos forneceram resultados satisfatórios.

De acordo com Kemp \& Kemp (1956), ao compararem estruturas com $10,5,2$ e 1 pontos, o número de amostras necessitou aumentar enquanto diminuia o número de pontos por amostragem. Já Rothery (1974), através de uma correlação teórica simples, demonstrou ser possível aumentar a precisão da es- 
timativa da cobertura vegetal, reduzindo o número de agulhas no aparato e mantendo o número de amostras.

\section{Parâmetros fitossociológicos}

Diversos parâmetros fitossociológicos foram sugeridos para a análise da vegetação com o uso do método de pontos, destacando-se aqui aqueles mais utilizados.

\section{- média de toques (MT)}

Corresponde a "cover repetition" de Goodall (1952) e indica o número médio de estratos da folhagem de uma espécie cobrindo o solo verticalmente. $\begin{array}{ll}\mathrm{MT}_{\mathrm{i}}=\mathrm{NT}_{\mathrm{i}} / \mathrm{NP}_{\mathrm{i}} & \mathrm{NT}_{\mathrm{i}}=\mathrm{N} \text { o de toques na espécie } \mathrm{i} \\ \mathrm{NP}_{\mathrm{i}}=\mathrm{N} \text { o de pontos com a espécie } \mathrm{i}\end{array}$

Goodall (1952) propôs o uso deste parâmetro no lugar da "relative frequency of each species in the cover" de Levy \& Madden (1933) porque este fornece um valor dependente da "percentage of ground covered by each species" ou freqüência absoluta.

- freqüência ou cobertura.

No método de pontos, o toque na agulha indica a cobertura do ponto por um ou mais indivíduos de uma ou mais espécies, daí poder-se denominar esse parâmetro de freqüência ou de cobertura, indiferentemente.

- freqüência ou cobertura absolutas (FA)

É a probabilidade de encontrar-se uma determinada espécie em 100 pontos, que é a unidade amostral adotada. Corresponde à "percentage of ground covered by each species" de Levy \& Madden (1933) e a "percentage cover" de Goodall (1952) e expressa a "frequency percentage" ou "frequency index" de Gleason (1920).

$\begin{array}{ll}F_{1}=100 . N_{i} / N T P & N P \\ \text { NTP }=\text { No to tal de pontos amostrados }\end{array}$

É um parâmetro que depende do padrão espacial, da densidade da espécie (Matteucci \& Colma, 1982), do número de pontos amostrados (MuellerDombois \& Ellenberg, 1974), da forma de vida, do tamanho dos indivíduos e da distância entre os pontos.

- freqüência ou cobertura relativas (FR)

É um valor comparativo das freqüências absolutas das espécies amostradas numa comunidade.

$\mathrm{FR}_{\mathrm{i}}=100 . \mathrm{FA}_{\mathrm{i}} / \Sigma \mathrm{FA}$ ou $\mathrm{FR}_{\mathrm{i}}=100 . \mathrm{NP}_{\mathrm{i}} / \Sigma \mathrm{NP}$

Para a análise fitossociológica de diferentes vegetações procura-se adequar o tamanho das parcelas ou a distância entre os pontos, para os métodos sem área, às formas de crescimento e tamanhos dos indivíduos, de maneira que estas características não interfiram na análise de freqüência. No método de pontos, por não haver possibilidade de adequação da amostra à vegetação, as 
formas de crescimento e os tamanhos dos indivíduos podem superestimar os valores obtidos para algumas espécies e subestimá-las para outras. Por isso, para Matteucci \& Colma (1982), o cálculo da freqüência ou cobertura relativa, com o uso do método de pontos não tem significado ecológico.

Além disso, parâmetros relativos fornecem informaçōes de valor limitado, podendo igualar comunidades ralas com comunidades densas (MuellerDombois \& Ellenberg, 1974).

Com todas as suas restrições, os dados de freqüência relativa expressam valores comparativos, isto é, situam a espécie dentro da vegetação, relacionando-a com as demais.

- freqüência ou cobertura na área (CR)

É a freqüência relativa analisada apenas nos pontos com ocorrência de espécies. Corresponde a "Percentage cover each species is contributing to the total area" de Levy \& Madden (1933).

$\mathrm{CR}=\left(100-\mathrm{N}_{\mathrm{O}}\right) \cdot \mathrm{FA}_{\mathrm{i}} / \Sigma \mathrm{FA}_{\mathrm{i}} \mathrm{N}_{\mathrm{O}}=$ Porcentagem de pontos sem toques

Equivale ao valor fornecido pela freqüência absoluta, dando valores semelhantes às espécies com igual ocorrência nas diferentes áreas, independente das demais, isto é, serve para a análise da espécie na área e não na vegetação.

Embora seus valores equivalham àqueles da freqüência absoluta, são calculados à partir dos valores de freqüência relativa, estando mais próximos destes quanto maior for a cobertura da vegetação na área.

Goodall (1952) sugeriu a exclusão deste parâmetro das análises por considerá-lo de pouco valor fitossociológico, não expressando nada que não possa ser melhor indicado pela porcentagem de solo descoberto e pela freqüência absoluta.

\section{- densidade}

Densidade é o número de indivíduos em uma área determinada. Como no método de pontos não há área e dificilmente ocorre mais de um indivíduo da mesma espécie em cada ponto, é um parâmetro que não possue expressão quando do uso deste método.

- vigor ou comportamento

Os termos vigor e comportamento estão sendo empregados como os definiram Matteucci \& Colma (1982), refletindo o êxito que uma espécie tem na comunidade.

- vigor absoluto (VA)

Corresponde a "relative frequency of each species in the cover" de Levy \& Madden (1933).
$\mathrm{VA}=100 . \mathrm{NT}_{\mathrm{i}} / \mathrm{NTP}$
$\mathrm{NT}_{\mathrm{i}}=\mathrm{N}$ - de toques na espécie $\mathrm{i}$
NTP $=$ № total de pontos amostrados

Este parâmetro reflete a estratificação ou a cobertura vertical de uma espécie e depende principalmente da sua forma de vida e desenvolvimento. É o valor, juntamente com o vigor relativo, que mais tem sido utilizado para a obtenção de relações com a biomassa 


\section{- vigor relativo (VR)}

Corresponde a "percentage each species is contributing to the pasture sward" de Levy \& Madden (1933) e a "percentage of sward" de Goodall (1952).

$\mathrm{VR}_{\mathrm{i}}=100 . \mathrm{VA}_{\mathrm{i}} / \Sigma \mathrm{VA}_{\mathrm{i}}$ ou

$\mathrm{VR}_{\mathrm{i}}=100 . \mathrm{NT}_{\mathrm{i}} / \Sigma \mathrm{NT}_{\mathrm{i}}=100 . \mathrm{NT}_{\mathrm{i}} / \mathrm{NTT} \quad \mathrm{NTT}=$ № total de toques

Da mesma forma que ocorre com os valores de freqüência, os dados de vigor relativo são maiores que os de vigor absoluto em áreas em que haja pouca estratificação da vegetação, isto é, onde o somatório dos vigores absolutos forem inferiores a $100 \%$ e, ao contrário, serão menores onde houver uma vegetação bastante estratificada.

- índice de valor de cobertura

Os índices compostos servem para indicar a importância da espécle na comunidade, sendo o mais conhecido deles o índice de valor de importância (IVI), de Curtis \& McIntosh (1950), definido como a soma da freqüência, densidade e dominância relativas (Whittaker, 1975).

Porque tanto a densidade quanto a freqüência são parâmetros que se relacionam com o número de indivíduos da população (Mueller-Dombois \& Ellenberg, 1974), e porque no método de pontos não há densidade, pode-se utilizar o índice de valor de cobertura, definido como a soma dos valores de freqüência e de dominância relativos.

Para os estudos de formações florestais, a área basal vem sendo usada como indicadora da dominância. Com o uso do método de pontos pode-se adotar, como valor de dominância, a biomassa, medida indiretamente pela "percentage each species is contributing to the pasture sward" de Levy \& Madden (1933) ou "percentage of sward" de Goodall (1952), aqui denominados de vigor.

- índice de cobertura(ic)

$O$ índice de cobertura é resultado da soma da freqüência absoluta, que indica a cobertura, com vigor absoluto, que pode ser correlacionado com a biomassa dos indivíduos de uma espécie.

$\mathrm{IC}_{\mathrm{i}}=\mathrm{FA}_{\mathrm{i}}+\mathrm{VA}_{\mathrm{i}}$

A comparação do valor obtido da somatória dos índices de cobertura de todas as espécies de uma comunidade com os valores obtidos de diversas comunidades poderá ser usada na discussão das densidades das vegetações(dv). Essa somatória poderá atingir valores variados.

$\mathrm{DV}=\Sigma \mathrm{IC}$ ou $\mathrm{DV}=\Sigma \mathrm{FA}_{\mathrm{i}}+\Sigma \mathrm{VA}_{\mathrm{i}}$

Os valores de $\Sigma \mathrm{FA}_{\mathrm{i}}$ e de $\Sigma \mathrm{VA}_{\mathrm{i}}$ indicarão se há maior contribuição da cobertura ou da estratificação das espécies.

Os valores de importância não dão idéia da biomassa ou da cobertura, que são considerados de maior significância ecológica que a densidade (Daunbenmire, 1968; Mueller-Dombois \& Ellenberg, 1974; Rice, 1967).

Os valores relativos servem para evidenciar as diferenças entre as espé- 
cies de uma comunidade e devem ser analisados com reservas na comparação entre comunidades.

O grande número de trabalhos efetuados com o método de pontos, dadas as variabilidades de aplicaçōes, aparatos, modificações e comparações que trazem, permite uma visão ampla das suas vantagens e limitações.

É um método que carece de estudos nas formações vegetais brasileiras e, mesmo, em condições de cultura ou outras alterações, que permitam dar-lhe embasamento para comparações e ampliação do uso.

Deve ser empregado com o uso de agulhas que simulem o ponto, de diâmetro o menor possível, isoladas, cuidando-se para sua disposição na vertical e mantendo-se padrões para comparação.

\section{Referências bibliográficas}

AHLGREN, H.L. 1947. A comparison of methods used in evaluating the results of pasture research. J.Am.Soc.Agron.14:1-99.

ARNBORG, T. 1949. Synpunkter pa vegetationsanalysen vid undersökningar av Nordiska Barrskogssamhällen. Svensk.Bot.Tidskrift 43:195-214.

ARNY, A.C. 1944. Alfafa and grass percentage determinations with the inclined point quadrat apparatus at different stages of development of mixtures. J.Am.Soc.Agron.36:996-8.

ARNY, A.C. \& A.R. SCHMID. 1942. A study of the inclined quadrat method of botanical analysis of pasture mixtures. J.Am.Soc.Agron.34:238-47.

BLACKMAN, G.E. 1935. A study by statistical methods of the distribution of species in grassland associations. Ann.Bot.49:749-77.

BOCHER, T.W. 1935. Om em metode til undersogelse af konstans skudtaethed og homogenitet. Bot.Tidsskr.43:278-304.

BOND, T.E.T. 1947. Some Ceylon examples of the logarithimic series and the index of diversity of plant and animal populations. Ceylon.J.Sci.A12:195-202.

BROEMBSEN, H.H. VON. 1965. A wheel-point apparatus for the survey and measurement of open and semi-open savannah vegetation. Proc.int.Grassl.Congr.9:1345-8.

BRUN, J.M. \& T.W. BOX. 1963. a comparison of line intercpts and random point frames for sampling desert shrub vegetation. J.Range Mgmt.16:21-5.

BUENO, O.L.; T.C. BUSELATO; S.T.S. MIOTTO. 1979. Composição florística de um campo localizado no município de Montenegro, Rio Grande do sul, Brasil. In: Anais Congresso Nacional Botânica. 30, Campo Grande. p. 153-8.

BUSELATO, T.C. \& O.L. BUENO. 1981. Composição florística de dois campos localizados no município de Montenegro, Rio Grande do sul, Brasil. Iheringia, Botânica, 26:65-84. 
CADWALLADR, D.A. \& J.V. MORLEY. 1973. Sheep grazing preferences on a salting pasture and their significance for wigean (Anas penelope L.) conservation. J.Br.Grassld.Soc.28:235-42.

CAIN, S.A. \& G.M.O. CASTRO. 1959. Manual of vegetation analysis. Harper \& Brothers, New York.

CASTELLANI, T.T. 1986. Sucessão secundária inicial em mata tropical semi-decídua, após perturbação por fogo. Unicamp, Campinas.

CHARPENTIER, C.A.G. \& O.SAARELA. 1941. Levy's pisteneliömenetelmä ja sen käyttö laindunnurmien kasvilliswistutkimurksissa. Valt. Maatalouskoet Julk.108:1-31.

COCKAINE, L. 1926. Tussock grassland investigations in New Zealand. In: A.G. TANSLEY \& T.F. CHIPP, eds.Aims and methods in the study of vegetation.Crow, London.

COOK, C.W. \& T.W. BOX. 1961. A comparison of the loop and point methods of analysing vegetation. J.Range Mgmt.14:22-7.

COUPLAND, R.T. 1950. Ecology of mixed prairie in Canadá. Ecol.Monogr.20:271-351.

CROCKER, R.L. \& N.S. TIVER. 1948. Survey methods in grassland ecology. J.Br.Grassld Soc.3:1-26.

CURTIS, J.T. \& R.P. MCINTOSH. 1950. The interrelations of certain analytic and synthetic phytosociological characters. Ecology 31:434-55.

DAUBENMIRE, R. 1968. Plant communities. Harper \& Row, New York. $300 \mathrm{p}$.

DAVIES, W. 1933. Methods of pasture analysis. I. The history of pasture analysis. Agric.Progr.10:225-31

DRE, W.B. 1944. Studies on the use of the point quadrat method of botanical analysis of mixed pasture vegetation. J.Agric.Res. 69:289-97.

DU TOIT, P.F. 1968. A simple method for the determination of leaf area of small leaves: S.Afr.J.Agric.Sci.11:201-2.

EDEN, T. \& T.E.T. BOND. 1945. The effect of manurial treatment on the growth of weed in tea. Empire J.Exp.Agric.13:141-57

ELLISON, L. 1942. A comparison of methods of quadratting shortgrass vegetation. J. Agric.Res.64:595-613.

EVANS, R.A. \& R. MERTON-LOVE. 1957. The step-point method of sampling. A practical tool in range research. J.Range Mgmt.10:208-12.

FABRIS, L.C.; O.J. PEREIRA; D.S.D. ARAUJO. 1990. Análise fitossociológica na formação pós-praia da restinga de Setiba, Guarapari - ES. In: Anais Simpósio Ecossistemas Costa Sul Sudeste Brasileiro: Estrutura, Função e Manejo. 2․ São Paulo. p.455-66.

FENTON, E.W. 1933. Methods of pasture analysis. V. The point quadrat method. Agric.Progr.10:238-42.

FLOYD, D.A. \& J.E. ANDERSON. 1987. A comparison of three methods for 
estimating plant cover. J.Ecol.75:221-8.

FORD, E.D. \& P.J. NEWBOLD. 1971. The leaf canopy of a coppiced deciduous woodland. J.Ecol.59:843.62.

FOWLER, G.W. \& L.G. ARVANITIS. 1981. Aspects of statistical bias due to the forest edge: Horizontal point samplin. Can.J.For.Res.11:334-41.

GARRISON, G.A. 1949. Uses and modifications of the point method for the "moosehorn" crown closure estimator. J.For.47:731-5.

GLEASON, H.A. 1920. Some applications of the quadrat method. Bull.Torrey Bot.Club 47:21-33.

GOODALL, D.W. 1952. Some considerations in the use of point quadrats for the analysis of vegetation. Aust.J.scient.Res.Ser.B.5:1-41.

GOODALL, D.W. 1953. Point quadrat methods for the analysis of vegetation. The treatment of data for tussok grass. Aust.J.Bot.1:457.61.

GREEN, K.M. 1978. Primate censusing in northern Colombia: A comparison of two techniques. Primates 19:537-50.

GREIG-SMITH, P. 1964. Quantitative plant ecology. 2a ed. Butterworths, London.

HANSON, H.C. 1934. A comparison of methods of botanical analysis of the native prairie in western North Dakota. J.Agric.Res.49:815-42.

HANSON, H.C. 1938. Ecology of grassland. Bot.Rev.4:51-82.

HANSON, H.C. 1950a. Ecology of grassland. II. Bot.Rev.16:283-360.

HANSON, H.C. 1950b. Vegetation and soil profiles in some solifluction and mound areas in Alaska. Ecology 31:606-30.

HEADY, H.F. 1957. The measurement and value of plant height in the study of herbaceous vegetation. Ecology 38:313-20.

HEADY, H.F. \& L. RADER. 1958. Modifications of the point frame. J.Range Mgmt.11:95-6.

HENSON, P.R. \& M.A. HEIN. 1941. A botanical and yield study of pasture mixtures at Beltsville, Maryland. J.Am.Soc.Agron.33:700-8.

HUGHES, E.E. 1962. Estimating herbage production using inclined point frame. J.Range Mgmt.15:323-4.

HURD, R.M. 1961. Grassland vegetation in the Big Horn mountains, Wyoming. Ecology 42:459-67.

IBRAHIM, K.M. 1971. Ocular point quadrat method. J.Range Mgmt.24:312.

JOHNSON, M.K. \& R.M. HANSEN 1977. Comparison of point frame and hand separation of coyote scats. J.Wildl.Mgmt.41:319-20.

JOHNSTON, A. 1957. A comparison of the line interception, vertical point quadrat and loop methods as used in measuring basal area of grassland vegetation. Can.J.Pl.Sci.37:34-42.

JULIN, E. 1948. Vesser udde mark och vegetation i en igenväxande lövand vid Bjärka-saby. Acta Phytogeogr.Suec.23:70-83.

KEMP, C.D. \& A.W. KEMP. 1956. The analysis of point quadrat area data. 
Aust.J.Bot.4:167-74.

KERSHAW, K.A. 1973. Quantitative and dynamic ecology. 2nd.ed. Edward Arnold, London. 183p.

KIM, C.M. \& H.S. LEE. 1975. Quantitative studies on the distribution of corticolous lichens in Korea. Korean N.J.Bot.18:38-44.

KLEINER, E.F. \& K.T. HARPER. 1972. Environment and community organization in grassland of Canyolands National Park. Ecology 53:299-309.

KUBÍKOVA, J. \& M. REJMÁNEK. 1973. Poznamky k nekterym Kvantitativnum metodám studia struktury rostlinnych spolecenstev. Preslia 45:154-64.

LEASURE, J.K. 1949. Determining the species composition of swards. Agron.J.41:204-6.

LEVY, E.B. 1927a. The grassland of New Zealand. Progress of regrassing experiments on deteriored hill county in Whangamomona County I. Interim results of burning ad sowing trials: seed mixture recommended for second burns. N.Z.J.Agric.34:145-64.

LEVY, E.B. 1927b. The grassland of New Zealand. Progress of regrassing experiments on deteriored hill county in Whangamomona County II. Studies on how best to win back secondary-growth contry. N.Z.J.Agric.35:361-75.

LEVY, E.B. \& W. DAVIES. 1929. Strain investigation relative to grasses and clovers. N.Z.J.Agric.34:1-8.

LEVY, E.B. \& W. DAVIES. 1930. Perennial rye grass strain investigations. N.Z.J.Agric.40:363-85.

LEVY, E.B. \& E.A. MADDEN. 1933. The point method of pasture analysis. N.Z.J.Agric.46:267-79.

LEVY, E.B. \& J.M. SMITH. 1929. Farmer's pasture competitions: North Taranaki results, 1928. N.Z.J.Agric.38:117-21.

LINDQUIST, B. 1931. Den Skandinaviska bokskogens biologi. Svenska Skogsv.Tidskr.29:179-532.

LOI, T.N. 1981. Environmental stresses and intertidal assemblages on hard substrates in the port of Long Beach, California, USA. Mar.Biol.63:197-212.

LONG, G.A.; P.S. POISSONET; J.A. POISSONET; P.M. DAGET; M.P. GODRON. 1972. Improved needled point frame exact line transects. J.Range Mgmt.25:228-9.

MACARTHUR, R.H. \& J.W. MACARTHUR. 1961. On bird species diversity. Ecology 42:594-8.

MAGAMBO, M.J.S. 1977. Canopy characteristics of seven clones of tea (Camelia sinensis L.) estimated by the use of point quadrat. Trop. Agric. 54:204-12.

MANTOVANI, W. 1987. Análise floristica e fitossociológica do estrado herbáceo-subarbustivo do cerrado na Reserva Biológica de Moji Guaçu 
e em Itirapina, $S P$. Unicamp, Campinas.

MATTEUCCI, S.D. \& A. COLMA. 1982. Metodologia para el estudio de la vegetacion. O.E.A., Washington.

MILLER, P.C. 1969. Tests of solar radiation models in three forest canopies. Ecology 50:878-85.

MORRISON, R.G. \& G.A. YARRANTON. 1970. An instrument for rapid and precise point sampling of vegetation. Can. J. Bot. 48:293-7.

MUELLER-DOMBOIS, D. \& H. ELLENBERG. 1974. Aims and methods of vegetation ecology. John Wiley \& Sons, New York.

MULLER, P.J. 1963. A stave-point apparatus for vegetation survey and measurement on steep and rock-strewn hill slopes. S. Afr. J. agric. Sci 6:339-44.

NERMEY, N.J. 1960. A modification for the point-frame method of sampling range vegetation. J. Range Mgmt. 13:261-2.

OSBORN, T.G.B.; J.G. WOOD; T.B. PALTRIDGE. 1935. On the climate and vegetation of Koonamore vegetation, Reserve to 1931. Proc. Linn. Soc. N.S.W. 60:392-427.

OWAGA, M.L.A. 1977. Comparison of analysis of stomach contents and faecal samples from zebra. East Afr. Wildl. J. 15:217-22.

PHILIP, J.R. 1965. The distribution of foliage denssity with foliage angle estimated from inclined point quadrat observations. Aust. J. Bot. 13:357-66.

PHILIP, J.R. 1966. The use of point quadrat with special reference to stem-like organs. Aust. J. Bot. 14:105-25.

POISSONET, P.S.; P.M. DAGET; J.A. POISSONET; G.A. LONG. 1972. Rapid point survey by bayonet blade. J. Range Mgmt. 25:313.

RADCLIFFE, J.E. \& N.S. MOUNTIER. 1964a. Problems in measuring pasture composition in the field. 1. Discu ssion on general problems and some considerations of the point method. N.Z.J. Bot. 2:90-7.

RADCLIFFE, J.E. \& N.S. MOUNTIER. 1964b. Problems in measuring pasture composition in the field. 2 . The effect of vegetation height using the point method. N.Z.J. Bot. 2:98-105.

RAUNKIAER, C. 1934. The life forms of plant and statistical geography. Clarendon, Oxford. 632p.

REPPERT, J.N.; M.J. MORRIS; C.A. GRAHAM. 1962. Estimation of herbage on California annual-type range. J. Range Mgmt. 15:318-23.

REYNOLDS, K.C. \& K. EDWARDS, 1977. A short focus telescope for ground cover estimation. Ecology 58:939-41.

RICE, E.L. 1967. A statistical method of determining quadrat size and adequacy of sampling. Ecology 48:1047-9.

RIPLEY, T.H.; F.M. JOHNSON; W.P. THOMAS. 1960. A useful device for sampling understory woody vegetation. J. Range Mgmt. 13:262-3.

ROBINSON, P. 1954. The distribution of plant population. Ann. Bot. (n.s.) 


\section{8:35-45.}

ROBINSON, P. 1955. The estimation of ground cover by the point quadrat method. Ann. Bot. (n.s.) 19:59-66.

ROTHERY, P. 1974. The number of pins in a point quadrat frame. J. Appl. Ecol. 11:745-54.

ROTHROCK, M.A. \& W.L. STERLING. 1982. Sequencial sampling for arthropodes cotton: Its advantages over point sampling. Southwestern Entomol. 7:70-81.

SCOTT, A.C. 1977. A review of the ecology of upper carboniferous plant assemblages, with new data from strathclyde. Paleontology 20:447-73.

SPRAGUE, V.G. \& W.M. MYERS. 1945. A comparative study of methods for determining yields of Kentucky blue grass and white clover when in association. J. Am. Soc. Agron. 37:370-7.

STANTON, F.W. 1960. Ocular point frame. J. Range Mgmt. 13:153.

TINNEY, F.W.; O.S. AAMODT; H.L. AHLGREN. 1937. Preliminary report of a study on methods used in botanical analysis of pasture swards. $J$. Am. Soc. Agron. 29:835-40.

TOMIALOJA, L. 1977. Bird census work in Poland. A progress report. Przegl. zool. 21:244-52.

VAN KEUREN, R.W. \& H.L. AHLGREN. 1957. A statistical study of several methods used in determining the botanical composition of a sward. I. A study of stablished pastures. Agron. J. 49:532-6.

WEST, O. 1937. An investigation of the methods of botanical analysis of pasture. S. Agr. J. Sci. 33:501-59.

WHITMAN, W.C. \& E.I. SIGGEIRSSON. 1954. Comparison of line interception and point contact methods in the analysis of mixed grass range vegetation. Ecology 35:431-6.

WHITTAKER, R.H. 1975. Communities and ecosystems. 2nd. ed. Macmilan, New York.

WILSON, J.W. 1959. Analysis of the spatial distribution of foliage by the two-dimensional point quadrats. New Phytol. 58:92-101.

WILSON, J.W. 1960. Inclined point quadrats. New Phytol. 59:1-8.

WILSON, J.W. 1963a. Estimation of foliage denseness and foliage angle by inclined point quadrats. Aust. J. Bot. 11:95-105.

WILSON, J.W. 1963b. Errors resulting from thickness of point quadrats. Aust. J. Bot. 11:178-88.

WINKWORTH, R.E. 1955. The use of point quadrats for the analysis of heathland. Aust. J. Bot. 3:68-81.

WINKWORTH, R.E. \& D.W. GOODALL. 1962. A crosswire sighting tube for point quadrat analysis. Ecology 43:342-3.

WINKWORTH, R.E.; R.A. PERRY; C.O. ROSSETTI. 1962. A comparison of methods of estimating plant cover in an arid grassland community. $J$. Range Mgmt. 15:194-6. 
YARRANTON, G.A. 1966. A plotless method of sampling vegetation. $J$. Ecol. 54:229-37. 